\title{
Toxic effects and DNA transformation
}

\author{
Jelenka Savkovic-Stevanovic
}

Faculty of Technology and Metallurgy, Belgrade University, Karnegijeva 4, 11000 Belgrade, Serbia.

Accepted 28 February, 2018

\begin{abstract}
In this paper, mutation point in the nucleotides transformations was studied. The major purine and pyrimidine bases were analyzed. Mutation are hereditary changes in genetic information, resulting from spontaneous or xenobiotic induced DNA damage. Point mutation and frame shift mutations were considered. Phosphorylation is the most important in the synthesis of purine and pyrimidine nucleosides and DNA damage. This paper considers impact of toxic effects to phosphorylation reactions modifications.
\end{abstract}

Keywords: Mutation, genes, DNA bases, chromosomes, mutation mechanism, metabolism.

E-mail: stevanoviccace@gmail.com.

\section{INTRODUCTION}

The term mutation can be applied to point mutations, which are qualitative changes involving one or a few bases within one gene, as well as to larger changes involving parts of chromosome detectable by light microscopy or even whole chromosomes and thus many thousand of genes (Michelson, 1963).

The structure and function of the purines and pyrimidines and their nucleosides and nucleotides have previously been studied in numerous literature (SavkovicStevanovic, 2009a, b). Synthetic analogs of naturally occurring nucleotides find application in cancer chemotherapy as enzyme inhibitors and can replace the naturally occurring nucleotides in nucleic acids (Ames et al., 1981).

Therapeutic attempts to inhibit the growth of cancer cells or certain viruses have often employed administration of analogs of bases, nucleosides, or nucleotides that inhibit the synthesis of either DNA or $R N A$. Allopurinol, a purine analog, is widely used in the treatment of gout (Prusoff and Ward, 1976).

In this paper point mutation was studied.

\section{DNA BASES}

The nucleotides participate in a wide variety of biochemical processes. Perhaps the best known role of purine and pyrmidine nucleotides is to serve as the monomeric precursors of RNA (ribonucleic acid) and $D N A$ (deoxyribonucleic acid). However, the purine ribonucleotides serve also as the ubiquitous high energy source, ATP (adenosine triphosphate), as regulatory signals (cycle AMP (adenosine monophosphate) [cAMP] and $G M P$ (guanine mnophosphate) [cGMP]), and as components of the coenzymes and of the methyl group donor adenosil methionine (Blum et al., 1990; Bishop, 1987, 1991). The pyrimidine nucleotides in addition to providing monomeric precursors for nucleic acid synthesis, also serve as high energy intermediates, such as UDP (uridine diphosphate) - glucose and UDPgalactose in carbohydrate metabolism and CDP (cytosine diphosphate)-acylglycerol in lipid synthesis.

The heterocyclic bases purine and pyrimidine are the parent molecules of nucleosides and nucleotides. Nucleotides are ubiquitous in living cells, where they perform numerous key functions (Savkovic-Stevanovic, 2010a, b). Examples include incorporation, as their ribose $(R N A)$ or deoxyribose (DNA) monophosphates, into nucleic acids, energy transduction $(A T P)$, parts of coenzymes $(A M P)$ acceptors for oxidative phosphorylation $(A D P)$ (adenine diphosphate) allosteric regulators of enzyme activity, and second messengers (cAMP), (cGMP) (Lewin, 1985).

Neither nucleotides nor their parent purine and pyrimidine bases in the diet are incorporated into human tissue nucleic acids or into purine or pyrimidine coenzymes (Henderson,1972). Even when a diet rich in nucleoproteins is ingested, human subjects form the constituents of tissue nucleic acids from amphibolic intermediates (Henderson and Paterson, 1973). This de 
novo synthesis permits purine and pyrimidine analogs with potential as anticancer drugs to be incorporated into $D N A$. The rates of synthesis of purine and pyrimidine oxy- and deoxyribonucleotides are subject to precise regulation (Hines et. al.,1994). Mechanisms have evolved to ensure production of these compounds in quantities and at times appropriate to meet varying physiologic demand (Michelson,1963).

In addition to de novo synthesis, these include "salvage" pathways for reutilization of purine or pyrimidine bases released by degradation of nucleic acids in vivo. Human diseases that involve abnormalities in purine or pyrimidine metabolism include gout, Lesch-Nyhan syndrome, Reye's syndrome, adenosine deaminase deficiency, and purine nucleoside phosphorylase deficiency (Prusoff and Ward, 1976).

\section{DNA AND RNA}

The metabolism of the purines and pyrimidines and their nucleosides and nucleotides has been investigated. The regulatory mechanisms of purine and pyrimidine biosynthesis were studied. The oligonucleotides hybridization and stacking interactions were examined (Savkovic-Stevanovic, 2010c).

Purine and pyrimidine bases that occur in the nucleotides are derived by substitution on the ring structures of the parent substances, purine and pyrimidine (Figures 1 to 3 ).

The three major pyrimidine bases present in the nucleotides of both procaryotes and eukaryotes are cytosine, thymine, and uracil. The purine bases adenine and guanine are the two major purines found in living organisms. Two other purine bases, hypoxanthine and xanthine, occurs as intermediates in the metabolism of adenine and guanine (Figures 1 and 2). In humans, a completely oxidised purine base, uric acid, is formed as the end product catabolism (Guengerich, 1991).

In nature, unusual bases occur in the addition to the 5 major described bases. Some of these unusual substituted bases are present only in the nucleic acids of bacteria and viruses, but many are also found in the DNA and transfer RNAs of both prokaryotes and eukaryotes (Cmarik et al., 1990). For example, both bacterial and human DNA contain significant quantities of 5methylcytosine, bacteriophages contain 5- hydroxylmethyl-cytosine. Unusual bases presenting the messenger RNA molecules of mammalian cells include $\mathrm{N}^{6}, \mathrm{~N}^{6}$-dimethyladenine, and $\mathrm{N}^{7}$-methylguanine. An uracil modified at the $\mathrm{N}_{3}$ position by the attachment of an $\alpha-$ amino, a-carboxyl-propyl group has also been detected in bacteria (Ziegler, 1988).

In plants, a series of purine bases containing methyl substituents occurs. Many have pharmacologic properties. Examples are coffee, which contains caffeine (1,3,7 -tri-methyl-xanthine), tea, which contains theophylline (1,3-di-methyl-xanthine) (Ziegler, 1988).
Guanine

(2-amino-6-oxypurine)

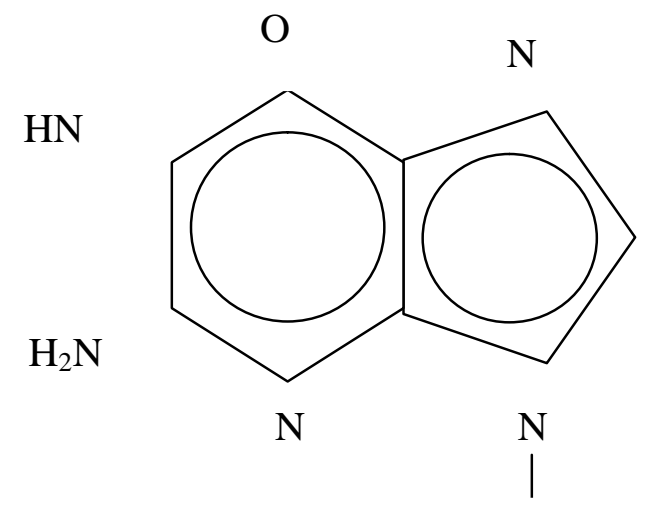

Adenine

$\mathrm{H}$

(6-aminopurine)

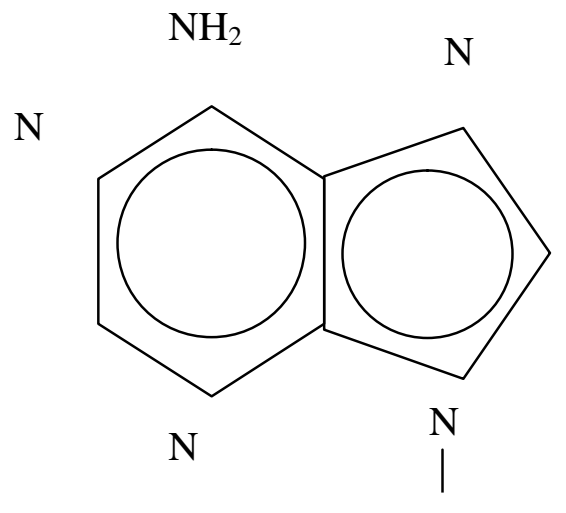

$\mathrm{H}$

Hypoxanthine

(6-oxypurine)

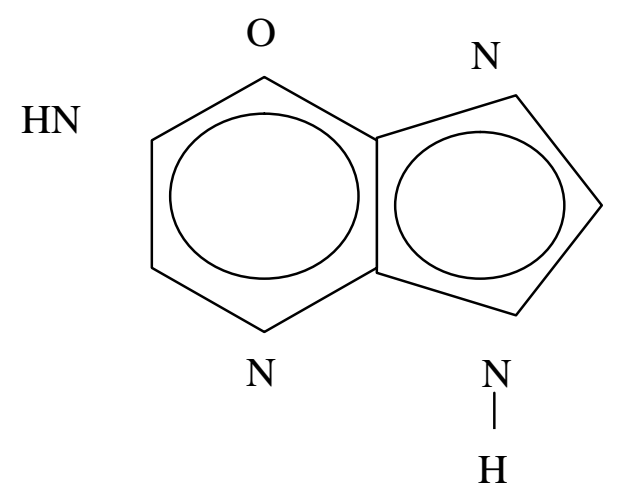

Figure 1. The major purine bases adenine, guanine, and hypoxanthine present in nucleotides.

Because of keto-enol tautomerism, several aromatic molecules can exist in a lactim or lactam form, the latter 
Xanthine

(2,6-dioxypurine)

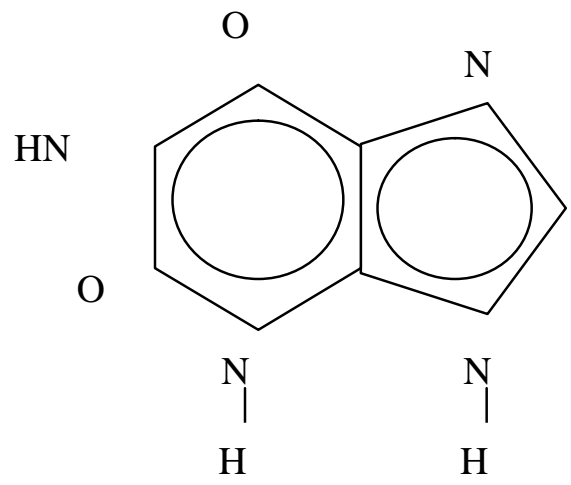

Figure 2. The major purine base present in nucleotides xanthine (2,6-dioxypurine).

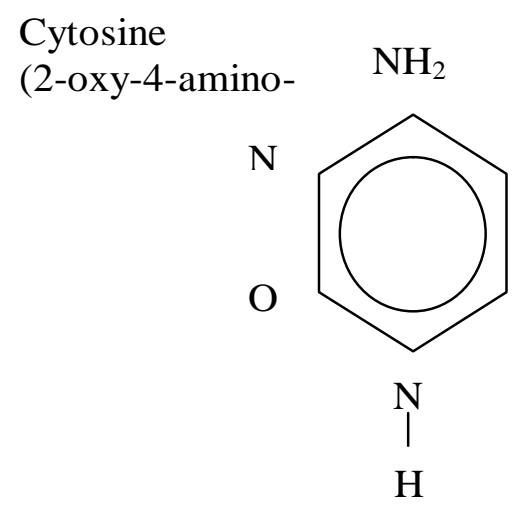

Thymine ( 2,4-dioxymethylpyrimidine)

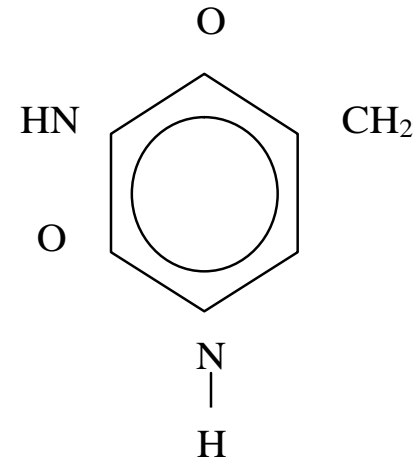

Figure 3. The major pyrimidine bases cytosine thymine present in nucleotides.

is by far the predominant tautomer of guanine or thymine under physiologic conditions (Martin and Gelfand, 1981).

At neutral $p H$, guanine is the least soluble of the bases, followed in the respect by xanthine. Although uric acids such as urate are relatively soluble at a neutral $p H$, it is highly insoluble in solutions with a lower $\mathrm{pH}$, such as urine. Guanine is not a normal constituent of human urine, but xanthine and uric acid do occur in human urine. These latter 2 purines frequently occur as constituents of urinary tract stones (Stanbury, 1983).

The free bases are less abundant in nature than their nucleosides and nucleotides. The nucleoside is composed of a purine or a pyramidine base to which a sugar (usually either D-ribose or 2-deoxyribose) is attached in $\beta$-linkage at $N_{9}$ or $N_{1}$, respectively. Thus, the adenine ribonucleside adenosine consists of adenine with D-ribose attached at $\mathrm{N}_{9}$. Guanosine consists of guanine with D-ribose attached at $\mathrm{N}_{9}$. Uridine is cytosine with ribose attached at its $\mathrm{N}_{1}$ position. Uridine consists of ribose attached at the position of uracil (Thelander and Reichard, 1979).

The 2'-deoxyribonucleosides consist of 2-deoxyribose attached to purine or pyrimidine bases. Attachment of the ribose or 2-deoxyribose to the ring structures is through glycosidic bond, which is relatively acid labile. Although, theoretically, free rotation of the sugar moiety and the purine or pyrimidine may occur (Smith, 1973).

A ring structure occurs about this $\mathrm{N}$-glycosidic bond, steric hindrance in fact hinders free rotation. The anti form is necessary for the proper positioning of the complementary purine and pyrimidine bases in the double stranded $\beta$-form of deoxyribonucleic acid (Thelander and Reichard, 1979).

Nucleotides are nucleosides phosphotylated on one or more of the hydroxyl groups of the sugar (ribose or deoxyribose). Thus, adenosine monophosphate (AMP or adenylate) is adenine + ribose + phosphate. 2'deoxyadenosine mono-phosphate (dAMP or deoxyadenylate) consists of 2-deoxyribose + phosphate. The only sugar commonly found attached to uracil is ribose, and that commonly found attached to thymine is 2- deoxyribose. Therefore, thymidic acid $(T M P)$ is thymine +2-deoxyribose + phosphate, and uridylic acid (UMP) is uracil +phosphate. DNA is a polymer of thymidilic acid, 2'-deoxycytidilic acid, 2'-deoxyadenylic acid, and 2'-deoxyguanylic acid. RNA is a polymer containing uridylate, citydilate, adenylate, and guanylate (Gusella,1986).

There are exemptions to the above structures of nucleotides. For example, in tRNA (transfer RNA) riboeis occasionally attached to uracil at the 5' position, establishing a carbon-to-carbon linkage instead of the usual nitrogen - to - carbon linkage. This unusually compound is called pseudo uridine $(\psi)$. tRNA molecules contain another unusual nucleotide structure, that is, thymine attached to ribose monophosphate. Pseudourydilic acid $(\psi M P)$ is similarly rearranged from urydilic acid after the tRNA molecule has been synthesized.

The abbreviations $A, G, C, T$, and $U$ may be used to designate the nucleosides that contain adenine, guanine, cytosine thymine, or uracil, respectively (Figure 1 to 3 ) 
(Prusoff and Ward, 1976). Because the phosphate are in the acid anhydride form a low entropy situation-the phosphates are said to be high energy ones, that is, high potential energy, the hydrolysis of $1 \mathrm{~mol} A T P$ to $A D P$ releases about $7 \times 4,16 \mathrm{KJ}$ of potential energy.

Free nucleotides also perform important functions in tissues.

The functional moieties of many vitamins are coenzyme nucleotides with structures analogous to purine and pyramidine nucleotides (Smith, 1973).

Mammals and most lower vertebrates are prototrophic for purines and pyrimidines, that is, they synthesize purine and pyrimidine nucleotides de novo.

Hypoxanthine ribonucleotide, usually called inosinic acid IMP or inosinate in the salt form, is a precursor of all purine ribonucleotides synthesized de novo.

Inosinate can also be formed by the deamination of $A M P$, a reaction which occurs particularly in muscle as part of the purine nucleotide cycle (Henderson, 1972). Inosinate, derived from $A M$, when reconverted to $A M P$ results in the net production of ammonia from aspartate. Removal of the phosphate group from IMP forms the nucleoside inosine (hypoxanthine riboside), an intermediate in another cycle referred to as the purine salvage cycle (Henderson and Paterson, 1973).

Inosine diphosphate IDP and inosine triphosphate ITP, analogs of $A D P$ and $A T P$ in which the purine nucleoside derivative is inosine rather than adenosine, occasionally participate in phosphorylation reactions (Prusoff and Ward, 1976).

Uridine nucleotide derivatives are important coenzymes in reactions involving the metabolism of hexoses and the polymerization of sugars to form starch and the oligosaccharide moieties of glycoproteins and proteoglycans. In these reactions, the substrates are uridine diphospho-sugars. For example uridine diphosphate glucose is the precursor of glycogen. Another uridine nucleotide coenzyme, uridine diphosphoglucuronic acid serves as the active glucuronide for conjugation reactions such as the formation of bilirubin glucuronide (Lewin, 1985).

Uracil also participates in the formation of high energy phosphate compounds analogous to ATP, GTP, or ITP. Uridine triphosphate, UTP is utilized for example, in the reactions involving conversion of galactose to glucose in which uridine diphosphate glucose and uridine diphosphate galactose also are formed. UTP is a precursor for the polymerization of uridine nucleotides into RNA (Gusella, 1986).

\section{MUTATION}

Point mutation is said to occur when one base is substituted for another (substitution) or when base pair is deleted or inserted (deletions/insertions) (Martin and Gelfand, 1981). Substitution of another purine for a purine base or of another pyrimidine for a pyrimidine base is called a transition. Substitution of purine for pyrimidine or pyrimidine for purine is called a transversion. Very slight alterations in the chemical structure of the DNA bases may be sufficient for a base pair substitution to occur. Guanine for example, normally pairs with cytosine, while $O^{6}$-methylguanine, frequent DNA modification seen with methylating agents such as dymethyl nitrosamine, pairs with tymine, resulting in a hereditary change of the genetic information (Lewin, 1985).

These changes in certain codons may cause insertion of the wrong amino acid into a relevant polypeptide. In this case, the changes are called missense mutation. Such proteins may have dramatically altered properties if the new amino acid is close to the active centre of an enzyme or affects the three dimensional structure of an enzyme or a structural protein (Blum, 1990).

Hence, the alternations may results in marked changes in the differentiations and proliferative characteristics of the affected cells. A base substitution can also result in the formation of a new inappropriate stop (or nonsense codon). The results of nonsense mutations are the formation of a shorter and, most likely, inactive protein. Owing to the redundancy of the genetic code, about a quarter of all possible base substitutions will not result in amino acid replacement and will be silent mutations (Bishop, 1991).

Bases can be also deleted or added to a gene. Because each gene has a precisely defined length, these changes, if they involve a number of bases that is not a multiple of three, result in a change in the reading frame of the DNA sequence and are known as frameshift mutations. Such mutations often have a dramatic effect on the polypeptide coded by the affected gene, because most amino acids will differ from the point of the insertion or deletion of bases in the DNA strand onward (Martin and Gelfand, 1981).

Some forms of unrepaired alkylated bases are lethal, due interference with DNA replication; others, such as $O^{6}$-methylguanine, lead to mutations if unprepared. These differences indicate that not all DNA adducts are of equivalent importance. In fact, some adducts appear not to interfere with normal DNA functions or to be rapidly repaired, others are mutagenic, and yet others are lethal. The most vulnerable base is guanine, which can form adducts at several of its atoms (N-7, C-8, O-6, and ecocyclic N-2) (Bock et al., 1990).

\section{METABOLISM}

Mammals and most lower vertebrates are prototrophic for purines and pyrimidines, that is, they synthesize purine and pyrimidine nucleotides via de novo pathway.

In human and other mammals, purine nucleotides are synthesized to meet the needs of the organism for the monomeric precursors of nucleic acids and for those 
other functions (Michelson, 1963).

The biosynthetic pathway for the synthesis of purine nucleotides can be summarized in the following steps. The first step in the synthesis of purine nucleotides is the formation of 5'-phosphoribosyl-1'-pyrophosphate (PRPP). The conversion of ribose 5-posphate and ATP to AMP $+P R P P$ is not however unique to the synthesis of purine nucleotides. PRPP also serves as a precursors of the pyrimidine nucleotides and required for the synthesis of $N A D$ and NADP (nicotinamide adenine dinucleotide phosphate), 2- coenzymes derived from niacin (Cmarik et al., 1990).

$P R P P$ then react with glutamine in a reaction catalyzed by phosphor ribosylpyrophosphate amidotransferase to form 5'-phosphoribosyl amine. The reaction is accompanied by the displacement of pyrophosphate and the formation of glutamate. Although other mechanism have been proposed for the synthesis of 5'phosphoribosylamine, the physiological important reaction in mammalian tissues is that catalyzed by the amidotransferase. 5'- phosphoribosylamine, then reacts with glycine to produce glycinamide ribosylphosphate. Synthesis of purine and pyramidine deoxyribonucleotides occurs by direct reduction at the 2'-carbon in the ribose moiety of the corresponding nucleotide, not by synthesis of the entire nucleotide utilizing 2'-deoxy analog of PRPP (Stanbury, 1983).

Several antimetabolites that are glutamine analogs are effective inhibitors of purine biosynthesis.

Conversion of $A M P$ and $G M P$ to their respective nucleoside diphosphates and nucleoside triphosphates occurs in 2 successive steps. The successive transfers of phosphate groups from ATP are catalysed by nucleoside monophosphate kinase and nucleoside diphosphate kinase, respectively. The enzyme that phosphorylates adenalyte is also called myokinase (Henderson and Paterson, 1973).

The pharmacologic approach has been to use an analog in which either the heterocyclic ring structure of the sugar moiety has been altered in such a way as to induce toxic effects when the analog becomes incorporated into various cellular constituents. Many of these effects results from the drug inhibition specific enzyme activities necessary for nucleic acids synthesis or from the incorporation of metabolites of the drug into the nucleic acids in which they alter the base pairing essential to accurate transfer of information (Hines et al., 1994).

\section{CONCLUSION}

In this study, mechanisms of muation and malignant transformation were investigated. The major purine and pyrimidine bases present in nucleotides were considered and the most vulnerable base is guanine, which can be affected during gene damage. Errors in substitution, deletion/insertion, transition, and transversion in mutations were studied.

DNA modification may cause insertion of the wrong amino acid into relevant polypeptides and these changes are called missense mutation.

\section{REFERENCES}

Ames BN, Cathcart R, Schwiers E, Hochstein P, 1981. Uric acid provides an antioxidant defense in humans against oxidant- and radical -caused aging and cancer: A hypothesis. Proc Natl Acad Sci, U.S.A., 78: 6858-6862.

Bishop JM, 1987. The molecular genetics of cancer. Science, 235: 305-311.

Bishop JM,1991. Molecular themes in oncogenesis. Cell, 64: 235-248.

Blum M, Grant DM, McBride W, Heim M, Meyer UA, 1990. Human arylamine $\mathrm{N}$-acetyltransferase genes: isolation, chromosomal localization, and functional expression. DNA Cell Biol, 9: 193-203.

Bock KW, Lipp HP, Block-Hening BS, 1990. Induction of drugmetabolizing enzymes by xenobiotics. Xenobiotica, 20: 1101-1111.

Cmarik JL, Inskeep PB, Meredith MJ, Meyer DJ, Ketterer B, Guengerich FP, 1990. Selectivity of rat and human glutathione-stransferase in activation of ethylene dibromide by glutathione conjugation and DNA binding and induction of unscheduled DNA synthesis in human hepatocytes. Cancer Res, 50: 2747-2752.

Guengerich FP, 1991. Reactions and significance of cytochrome P-450 enzymes. J Biol Chem, 266: 10019-10022.

Gusella JF, 1986. Recombinant DNA techniques in the diagnosis and treatment of inherited disorders. J Clin Invest, 77: 1723-1726.

Henderson JF, 1972. Regulation of purine biosynthesis, Monograph, No 170, American Chemical Society.

Henderson JF, Paterson ARP, 1973. Nucleotide metabolism: An introduction. Academic Press.

Hines RN, Cashman JR, Philpot RM, Williams DE, Ziegler DM, 1994. The mammalian flavin-containing monooxygenases: Molecular characterization and regulation of expression. Toxicol Appl Pharmacol, 125: 1-6.

Lewin B, 1985. Genes II. J. Wiley, New York.

Martin DW Jr, Gelfand EW, 1981. Biochemistry of diseases of imunodevelopment. Ann Rev Biochem, 50: 845.

Michelson AM, 1963. The chemistry of nucleosides and nucleotides. Academic Press.

Prusoff WH, Ward DC, 1976. Nucleoside analogs with antiviral activity. Biochem Pharmacol, 25: 1233-1239.

Savkovic-Stevanovic J, 2009a. Human tissue information processing. WSEAS, $2^{\text {nd }}$ International Conference Biomedical Electronics and Biomedical Informatics, Moscow, Russia, August 20-22, pp.64-69

Savkovic-Stevanovic J, 2009b. Informational macromolecule in biological systems, MCBC2009-Mathematics and Computers in Biology and Chemistry, Prague, March, 23-25, 2009.

Savkovic-Stevanovic J, 2010a. Nucleotides recombination, state. Trans Biol Biomed, 7(3): 253-262.

Savkovic-Stevanovic J, 2010b. DNA combination and recombination state. 10th International Conference on Mathematics and Computers in Biology and Chemistry. ID632-188, Iashi, 11-12 June, 2010.

Savković-Stevanović J, 2010c. DNA hybridization channel fundamentals. The 7th WSEAS International Conference on Mathematical Biology and Ecology-MABE2010. pp. 469-474. Coimbatore, India, February 18-20.

Smith LH Jr, 1973. Pyrimidine metabolism in man. N Engl J Med, 288: 764-771.

Stanbury JB, Wyngaarden JB, Fredrickson DS, 1983. The Metabolic Bases of Inherited Disease. $5^{\text {th }}$ ed., McGraw Hill.

Thelander L, Reichard P, 1979. Reduction of ribonucleotides. Annu Rev Biochem, 48: 133-158.

Ziegler DM, 1988. Flavin-containing monooxygenases: catalytic mechanism and substrate specificities. Drug Metab Rev, 19: 1-32.

Citation: Savkovic-Stevanovic J, 2018. Toxic effects and DNA transformation. Biochem Biotechnol Res, 6(2): 15-19. 